\title{
The influence of training and experience on memory strategy
}

\author{
John Patrick • Phillip L. Morgan • Victoria Smy • \\ Leyanne Tiley • Helen Seeby • Tanya Patrick • \\ Jonathan Evans
}

Published online: 16 January 2015

(C) Psychonomic Society, Inc. 2015

\begin{abstract}
This paper investigates whether, and if so how much, prior training and experience overwrite the influence of the constraints of the task environment on strategy deployment. This evidence is relevant to the theory of soft constraints that focuses on the role of constraints in the task environment (Gray, Simms, Fu, \& Schoelles, Psychological Review, 113: 461-482, 2006). The theory explains how an increase in the cost of accessing information induces a more memory-based strategy involving more encoding and planning. Experiments 1 and 3 adopt a traditional training and transfer design using the Blocks World Task in which participants were exposed to training trials involving a 2.5 -s delay in accessing goal-state information before encountering transfer trials in which there was no access delay. The effect of prior training was assessed by the degree of memory-based strategy adopted in the transfer trials. Training with an access delay had a substantial carryover effect and increased the subsequent degree of memorybased strategy adopted in the transfer environment. However, such effects do not necessarily occur if goal-state access cost in training is less costly than in transfer trials (Experiment 2). Experiment 4 used a fine-grained intra-trial design to examine the effect of experiencing access cost on one, two, or three occasions within the same trial and found that such experience on two consecutive occasions was sufficient to induce a more memory-based strategy. This paper establishes some effects of training that are relevant to the soft constraints theory and also discusses practical implications.
\end{abstract}

J. Patrick $(\square) \cdot$ V. Smy $\cdot$ L. Tiley $\cdot$ H. Seeby $\cdot$ T. Patrick $\cdot$ J. Evans School of Psychology, Cardiff University, Cardiff CF10 3AT, UK e-mail: PatrickJ@cardiff.ac.uk

P. L. Morgan

Department of Health and Social Sciences, University of the West of England, Frenchay Campus, Bristol BS16 1QY, UK
Keywords Training $\cdot$ Experience $\cdot$ Strategy $\cdot$ Access cost . Soft constraints $\cdot$ Transfer

The strategy used to perform any task may determine not only the efficacy of performance but also the extent of the cognitive resources required. In most tasks, different strategies are available to achieve the same goal and there are various well documented factors that affect choice of strategy, including degree of cognitive processing (e.g., Lemaire \& Lecacheur, 2010; Luwel, Lemaire, \& Verschaffel, 2005; Luwel, Schillemans, Onghena, \& Verschaffel, 2009), history of success using a strategy (e.g., Lovett \& Anderson, 1996; Siegler \& Shipley, 1995), the similarity of the task to other tasks (e.g., Gick \& Holyoak, 1987; Thorndike \& Woodworth, 1901), and individual factors such as level of skill and working memory capacity (e.g., Campbell \& Xue, 2001; LeFevre, Bisanz, Daley, Buffone, Greenham, \& Sadesky, 1996). An important perspective on strategy selection comes from ACT-R theorists (e.g., Anderson \& Lebiere, 1998) who emphasize that cognition is adaptive to the environment and strategy selection involves a cost-benefit tradeoff. In the same tradition is the theory of soft constraints (Gray \& Fu, 2004; Gray, Simms, Fu, \& Schoelles, 2006), which elaborates how the degree of memory-based strategy utilized to perform a task adapts to changes in the constraints of the task environment. Increasing the cost of accessing information within a task will induce a more memory-based strategy compared to when such information is available without such an access cost (Fu \& Gray, 2000; Gray \& Fu, 2004; Gray et al., 2006; Morgan, Patrick, Waldron, King, \& Patrick, 2009; Morgan, Patrick, \& Tiley, 2013). The theory of soft constraints acknowledges that training and/or top-down demands can overwrite the effect of the task environment on the degree of memory-based strategy that is selected (Gray et al., 2006), although to the best of our 
knowledge there is no empirical evidence bearing on this theoretically important issue. Therefore the main aim of this paper is to investigate whether previous training and experience can overwrite the subsequent influence of the constraints of the task environment on strategy deployment and would therefore need to be taken into account by the theory.

First, we briefly review the theory of soft constraints (Gray et al., 2006). Second, we discuss why prior training or experience may affect subsequent strategy deployment.

\section{The theory of soft constraints}

The theory of soft constraints (Gray \& Fu, 2004; Gray et al., 2006) follows in the tradition of research and theory on rational behavior (e.g., Anderson, 1990; Chater \& Oaksford, 1999). It focuses on the selection of low-level task strategies made up of perceptual, cognitive, and motor elements that occur in, for example, human computer interaction. Gray et al. (2006) proposed that a task is made up of "hard" and "soft" constraints. Hard constraints dictate what behaviours are or are not possible when performing a task, whilst soft constraints are flexible and concern how the person tackles the task, including the strategy chosen. Strategy selection is driven by the perceived cost-benefit balance and the need to minimize cost, particularly local time cost (Gray et al., 2006), even at the millisecond level of task performance (Gray \& Boehm-Davis, 2000). Hence strategy selection can be influenced by imposing a small time delay in accessing information (a hard constraint) that increases the degree of memorybased strategy adopted (a soft constraint). This strategy shift occurs because participants find it beneficial to increase encoding and planning rather than paying the access cost of a brief time delay on each occasion the information is viewed (Gray et al., 2006). Therefore, somewhat counter-intuitively, increasing the time associated with accessing information can induce the deployment of a more memory-based strategy that involves greater encoding and planning. Hence cognition adapts to encode more chunks of information in internal memory rather than relying so much on the display as an external memory resource and paying a time cost on each occasion it is consulted. Originally Fu and Gray (2000) used the Blocks World Task (BWT), developed by Ballard, Heyhoe, and Pelz (1995), in order to study the effects of varying information access cost. The BWT used in the present study is a visuo-spatial copying task, and access cost was increased by imposing a time delay of a few seconds before a participant was able to view the target pattern of colored blocks to be copied (see the "Method" section of Experiment 1).

There is now considerable evidence supporting the prediction of the theory of soft constraints that increased access cost will encourage a more memory-based strategy. If the cost of accessing information is perceived to be low then a lower memory-based strategy is adopted and the display is used more as an external memory resource (e.g., Fu \& Gray, 2000; Gray \& Fu, 2004; Gray et al., 2006; Morgan et al., 2009; Morgan \& Patrick, 2013). A shift to a more memorybased strategy can be induced by a relatively small access cost of a couple of seconds. In Experiment 3 by Gray et al. (2006), a range of increasing access costs from 0 to $3.2 \mathrm{~s}$ were used that resulted in a linear increase in the degree of memorybased strategy deployed, accounting for $97 \%$ of the variation among access-cost conditions. In the present experiments we used an access time delay of $2.5 \mathrm{~s}$ as this value should induce a shift in the degree of memory-based strategy adopted by participants during training trials.

Inducing a more memory-based strategy by increased access cost can: (a) mitigate the negative effects of interruption by reducing forgetting post-interruption (e.g., Morgan et al., 2009); (b) encourage better use of an interruption lag, which is a short delay before an interrupting task that provides an opportunity to encode suspended goals for retrieval postinterruption (Morgan et al., 2013); and (c) also encourage better planning and solution efficiency in problem solving (Morgan and Patrick, 2013). The focus of the theory of soft constraints (Gray et al., 2006) is on the effect of constraints of the task environment on performance and is consistent with a wide-ranging literature suggesting that many aspects of behavior are controlled by short-term rather than long-term tradeoffs (e.g., Hernstein \& Vaughn, 1980; Loewenstein, Read, \& Baumeister, 2003). The theory acknowledges that short-term cost-benefit considerations may be overridden by factors such as training. Therefore the aim of the present paper is to investigate whether adaptation to the hard constraints of the task environment is affected by prior training in an environment with different constraints. This is theoretically important and below we review reasons why prior training or experience may affect the subsequent degree of memory-based strategy deployed.

\section{The influence of training and experience}

History of success at using a particular strategy to perform a task is one indicator of whether people will persist with the same strategy (Luchins 1942; Reder, 1987), and this factor is identified as important in ACT-R theory (Anderson, Bothell, Byrne, Douglass, Lebiere, \& Qin, 2004). For example, Lovett and Anderson (1996) developed the Building Sticks Task and found that if a particular operator was used successfully in the past, then it was more likely to be used again. However, there are many examples in the literature of a previously successful strategy persisting even when another may be more efficient in terms of speed and/or accuracy (e.g., Luwel, Vershaffel, Onghena, \& De Corte, 2003). The classic illustration of this phenomenon comes from the water jar studies carried out by 
Luchins (Luchins 1942; Luchins \& Luchins, 1959) involving what he termed the Einstellung effect. In these studies participants were exposed to as few as five training problems that were soluble by only one problem-solving strategy. It was found that participants persisted in using this strategy with subsequent problems even though a more efficient alternative strategy was available. This phenomenon has also been labeled the "Paradox of the Active User" by Carroll and Rosson (1987) and further discussed by Fu and Gray (2004).

It is important to note that what constitutes strategy selection in the studies by Luchins (1942), Lemaire and Lecacheur (2010), and Luwel et al. (2003) differs from that of the present study. In the former studies, strategy selection was defined by a choice of operators and how they were organized in performing the task, whereas in the present study the operators remain the same but what is selected is the degree of memorybased strategy adopted. Consequently whilst the BWT involves elements of perception, planning, encoding, retrieval, and motor output, it is the extent of the cognitive processing of these elements that defines the degree of memory-based strategy. Therefore strategy selection differences in the current study concern quantitative differences in cognitive processing rather than qualitative differences in strategy, defined by the selection of different operators. A further difference is that neither a higher nor a lower level of memory-based strategy can be characterized as better at carrying out the BWT as both can result in successful task completion.

Another relevant research area concerns the cost of strategy switching (Lemaire \& Lecacheur, 2010; Luwel et al., 2009), although, in the present study, the only strategy switch is in the degree of memory-based processing. However, if strategy switching involves some cognitive cost, then this may inhibit any switch from a previous strategy to one that is more congruent with the present task environment. This could be a contributing factor that explains why participants in Luchins' experiments failed to utilize a more efficient strategy that was available after training. Lemaire and Lecacheur (2010) investigated the utilization of different arithmetic strategies over a series of trials that involved both instructions to switch and voluntary switching. In both situations participants preferred not to switch strategies between trials, and when they did, performance was worse, particularly with the easier task and strategy. Similarly Luwel et al. (2009) found that when participants were given a choice over strategy, they favored using the same strategy over two consecutive problems rather than fluctuating between different strategies.

Some explanations of switch cost are borrowed from the task switching literature (e.g., Allport, Styles, \& Hsieh, 1994; Altmann \& Gray, 2008; Monsell, 2003). One view is that a previously employed strategy will be at a higher level of activation and therefore its retrieval will be easier than one not used recently (e.g., Allport et al., 1994). Alternatively, some reconfiguration of cognitive resources may be necessary in order to move from selection and execution of a previous strategy to a different current one (Rogers \& Monsell, 1995). Both explanations involve increased cognitive processing that may act to discourage a switch to a second strategy and these cognitive costs would be expected to increase with the number of trials on which the previous strategy is executed.

Finally, a traditional area that highlights the importance of prior experience upon performance concerns transfer of knowledge and skill (e.g., De Corte, 2003; Reder \& Klatzky, 1994). The theory of identical elements, originally proposed by Thorndike and Woodworth (1901) with subsequent modifications by Osgood (1949), Gick and Holyoak (1987), and Anderson (1987), emphasizes that positive transfer will increase the greater the similarity between the tasks and skills involved in the training and transfer situations. This is consistent with the notion of transfer being determined by the degree of transfer-appropriate processing (e.g., Franks, Bilbrey, Lien, \& McNamara, 2000; Morris, Bransford, \& Franks 1977; Roediger, Gallo, \& Geraci, 2002). More recently, a variant of this has been termed the "procedural reinstatement principle" (e.g., Clawson, Healy, Ericsson, \& Bourne Jr, 2001; Healy, Wohldmann, Sutton \& Bourne Jr, 2006). According to this principle, an individual will show high durability and positive transfer during skill testing only when the mental procedures developed during training can be reinstated at the time of testing.

In summary, these different literatures are consistent in suggesting that prior experience or training can influence subsequent task performance. Experiments 1-3 investigate the influence of training in one task environment on the subsequent degree of memory-based strategy deployed in a subsequent transfer environment. More specifically we vary the access cost between these two environments in order to assess whether, and if so how much, training with one level of access cost will influence subsequent behavior in an environment with a different access cost.

\section{Experiment 1}

Experiment 1 aimed to investigate the influence of training in a high access-cost environment, which induces a high memory-based strategy, on subsequent performance in a medium access-cost environment, which would normally be congruent with a lower memory-based strategy. A carry-over effect was predicted for various reasons. From the perspective of the soft constraints theory (Gray et al., 2006), there is no additional cost in persevering with the higher memory-based strategy, and the elimination of the access cost in the medium access transfer environment improves the cost-benefit tradeoff of this strategy. Therefore persisting with a previously successful strategy when the cost-benefit tradeoff becomes more attractive represents a rational approach. Also, by maintaining 
the higher memory-based strategy, there is no cognitive cost from switching the degree of memory-based strategy, even though memory load could be reduced with a lower memory-based strategy.

\section{Method}

\section{Participants}

Sixty-six Cardiff University students were randomly assigned to one of three conditions, 48 women and 18 men with an age range of $18-45$ years $(M=20.89, S D=4.47)$. Studies of high access cost (e.g., Morgan et al., 2009, Experiment 1) reported large effect sizes $(f=.76-.97)$ on strategy measures. Statistical power for all four experiments was calculated using G*Power 3.1.7 software (Faul, Erdfelder, Lang, \& Buchner, 2007; Faul, Erdfelder, Buchner, \& Lang, 2009). The sample size was adequate to detect a large effect size $(f \geq .4)$ with power of .8 .

\section{Materials}

The BWT is a visuo-spatial copying task (see Fig. 1) in which the goal is to copy a pattern of ten colored blocks in a $4 \times 4$ target grid (Fig. 1, top left) to a workspace window (top right) by dragging blocks, one at a time, from a resource window (bottom left). In some conditions the target window is covered by a grey mask that disappears when the participant moves their mouse cursor into the target window but reappears when it moves out of this window. Therefore, in this condition when the mask covering the goal state is removed, a plan has to be generated and encoded concerning what blocks should be moved and to what positions. This plan has to be executed by moving these blocks from the resource window to the workspace window while the goal state is occluded. The target pattern constitutes ten colored blocks and six empty/grey spaces with no color used twice, and a different randomized target pattern was used for each BWT problem. All mouse movements and copying activity were recorded and time stamped at the millisecond level of measurement.

\section{Design}

There were three training conditions: High and Medium access-cost conditions; and a no-training control condition. In the Medium access-cost condition, the target pattern was covered with a mask that disappeared immediately when the mouse cursor was moved into the target window and reappeared when it was moved out of the target window. The same occurred in the High access-cost condition except that there was an extra 2.5-s lockout time for the mask to disappear from the target window when the mouse cursor was in the target window. Participants in both access-cost training conditions received 30 training trials whereas those in the no training control condition received none. In the following transfer phase, all three conditions received ten transfer trials at medium access cost. There was no gap between the training and transfer trials and participants were not informed of any change of environment, so that the degree of memorybased strategy adopted in both training and transfer environments was spontaneously adopted and not a result of briefing and anticipation.

Three measures were used to determine the degree of memory-based strategy adopted by participants across the transfer phase. The first measure is the number of blocks copied correctly after the first visit to the target window, which $\mathrm{Fu}$ and Gray (2000) have cited as the most important indicator of the degree of memory-based strategy. This is because it encompasses the nature of all strategy elements from perceptual

\begin{tabular}{|l|l|l|l|}
\hline Salmon & & $\begin{array}{l}\text { Dark } \\
\text { Blue }\end{array}$ & Violet \\
\hline & Pink & Yellow & \\
\hline Red & & & $\begin{array}{l}\text { Light } \\
\text { Blue }\end{array}$ \\
\hline & Green & Black & White \\
\hline
\end{tabular}

\begin{tabular}{|l|l|l|l|}
\hline & Red & White \\
\hline & $\begin{array}{l}\text { Light } \\
\text { Blue }\end{array}$ & Yellow & $\begin{array}{c}\text { Dark } \\
\text { Blue }\end{array}$ \\
\hline Violet & & & Salmon \\
\hline & Black & Green & Pink \\
\hline
\end{tabular}

\begin{tabular}{|c|c|c|c|c|c|}
\hline Salmon & Green & White & Violet & Yellow & \\
\hline Red & Black & $\begin{array}{l}\text { Dark } \\
\text { Blue }\end{array}$ & Pink & $\begin{array}{l}\text { Light } \\
\text { Blue }\end{array}$ & \\
\hline
\end{tabular}

\section{Stop trial}

Fig. 1 Example of the Blocks World Task (Experiments 1-3). Note. Words are used in this figure to indicate actual colors used 
input to motor output, and is uncontaminated by any previous behavior in the trial that may vary among conditions. The more blocks copied correctly, the higher the memory-based strategy deployed. The second measure is the average time spent during the first target window visit, which focuses on the input strategy elements of perception, encoding, and planning. Presumably, as this time increases, more blocks are perceived and more attributes concerning their position and color are selected and encoded. Finally, the average number of visits to the target window is a more global reflection of strategy that takes account of behavior across the whole trial.

A higher memory-based strategy is demonstrated by more blocks copied correctly after the first visit to the target window, and more time spent there encoding and planning moves, thus requiring fewer overall visits to view the target pattern. However, a higher memory-based strategy may be accompanied by more errors. Therefore we also collected data concerning the number of blocks incorrectly placed after the first visit to the target window and the number of occasions the participant incorrectly pressed the stop trial button when the target pattern was incomplete.

\section{Procedure}

Participants were tested individually and instructed how to copy and move blocks within the BWT but were not instructed in any strategy or any performance criterion such as speed or accuracy. When participants believed that the target pattern had been successfully copied to the workspace window, they pressed a "stop trial" button (Fig. 1). If an error had been made, participants were required to revise their pattern before the next trial. The Experiment lasted between 15 and 35 minutes, depending on the condition.

\section{Results and discussion}

If differences exist in the degree of memory-based strategy among the training conditions in the transfer environment, then we can infer that the medium access cost of the transfer environment did not fully control strategy deployment and that prior training had some carry-over effect. Therefore, our initial analyses examined differences among training conditions on the transfer trials. All outliers with z-scores higher than 3.29 were replaced with the grand mean (Field, 2013).

Effect of training on the degree of memory-based strategy adopted at transfer

The degree of memory-based strategy exhibited by participants in the transfer phase increased on all three strategy measures as access cost increased among the three conditions
(Table 1). One-way ANOVAs confirmed significant main effects of training condition on: number of blocks copied correctly after the first visit to the target window, $F(2,63)=$ $10.02, M S E=.59, p<.001, f=.56$; average time spent during the first visit, $F(2,63)=7.03, M S E=2.77, p<.01, f=.47$; and number of visits to the target window, $F(2,63)=19.50, M S E$ $=2.42, p<.001, f=.79$. Bonferroni post-hoc comparisons revealed that participants in the High access-cost training condition, compared to those in the Medium access-cost and notraining conditions, copied significantly more blocks correctly after the first target visit ( $p s<.01$ and .001 respectively), spent more time on average in the target window during the first visit ( $p s<.05$ and .01 respectively) and made fewer visits to the target window $(p s<.001)$. Therefore performance on all three measures consistently indicates that a more memorybased strategy was adopted in the transfer environment after High access-cost training whereas the Medium access-cost training had no effect on transfer as it did not differ on any of the three measures from the condition with no training. Given the lack of any effect of Medium access-cost training on transfer, we can conclude that the transfer effect of the High access-cost condition was totally due to the 2.5 -s lockout time delay.

Also, and not surprisingly, there were strong positive relationships between the time spent encoding and planning in the target window during the first visit and the subsequent number of blocks copied correctly for the High $(r(22)=.67, p<.001)$, Medium $(r(22)=.73, p<.001)$, and control $(r(22)=.74, p<$ $.001)$ conditions. Consequently, as encoding time increases so does the number of blocks correctly copied for all conditions, and the effect of increasing access cost is to induce more time encoding and planning when viewing the target pattern.

Three one-way ANOVAs examined whether there was any dissipation in the carry-over effect across the ten transfer trials in the degree of memory-based strategy used by participants in the High access-cost training. There were no significant differences across the transfer trials in the number of blocks copied correctly after the first visit to the target window, $F(9,189)=.64, M S E=$ $2.70, p=.76$; average time spent during the first visit to the target window, $F(15.22,109.63)=1.69, M S E=$ $11.48, p=.14$, using the Huynh-Feldt correction due to violation of sphericity; and number of visits to the target window, $F(9,189)=1.01, M S E=3.25, p=.43$. Hence, there is no evidence of the effect of High access training dissipating across the ten transfer trials.

Finally, the average error rate across all conditions for blocks incorrectly placed after the first target visit was $8.48 \%$ and for the number of trials incorrectly stopped was $7.73 \%$. Out of the participants who committed errors, there were no differences in the number of errors committed between the three conditions on either error measure, $F(2,31)=1.99, M S E=.02, p=$ 
Table 1 The effect of training on transfer performance (Experiment 1)

\begin{tabular}{|c|c|c|c|c|}
\hline \multirow{2}{*}{$\begin{array}{l}\text { Measures of degree of memory-based strategy } \\
\text { (across ten transfer trials) }\end{array}$} & & \multicolumn{3}{|l|}{ Training condition } \\
\hline & & High access-cost $(n=22)$ & Medium access-cost $(n=22)$ & No-training (control) $(\mathrm{n}=22)$ \\
\hline $\begin{array}{l}\text { Number of blocks copied correctly after the first visit } \\
\text { to the target window }\end{array}$ & $\begin{array}{l}M \\
S D\end{array}$ & $\begin{array}{l}2.85 \\
0.92\end{array}$ & $\begin{array}{l}2.14 \\
0.71\end{array}$ & $\begin{array}{l}1.85 \\
0.66\end{array}$ \\
\hline Time spent during the first visit to the target window (s) & $\begin{array}{l}M \\
S D\end{array}$ & $\begin{array}{l}3.96 \\
2.14\end{array}$ & $\begin{array}{l}2.65 \\
1.70\end{array}$ & $\begin{array}{l}2.14 \\
0.91\end{array}$ \\
\hline Number of visits to the target window per trial & $\begin{array}{l}M \\
S D\end{array}$ & $\begin{array}{l}4.41 \\
1.02\end{array}$ & $\begin{array}{l}6.44 \\
1.93\end{array}$ & $\begin{array}{l}7.25 \\
1.58\end{array}$ \\
\hline
\end{tabular}

.15 , and, $F(2,27)=.83, M S E=0.01, p=.45$ respectively. Consequently there is no evidence that the higher memory-based strategy adopted is associated with a higher error rate.

The relative contributions of training and transfer environments to strategy

Another aim was to calibrate the relative contributions of the High and Medium access-cost training to the degree of memory-based strategy utilized during transfer. There was a reduction in the average number of blocks copied between the training $(M=3.45)$ and transfer $(M=2.86)$ environments by participants in High access training but, importantly, this did not drop to the level of performance in the transfer environment of those in the Medium access-cost condition (Fig. 2). In contrast, as expected, there was no difference in blocks copied between training $(M=2.15)$ and transfer $(M=2.14)$ environments for those in the Medium access training condition. A 2 (condition: High and Medium access cost) $\times 2$ (environment: training and transfer) mixed ANOVA confirmed an interaction between these two variables, $F(1,42)=8.24, M S E=.24, p<.01, f=$ .44. In the High access-cost condition there was a

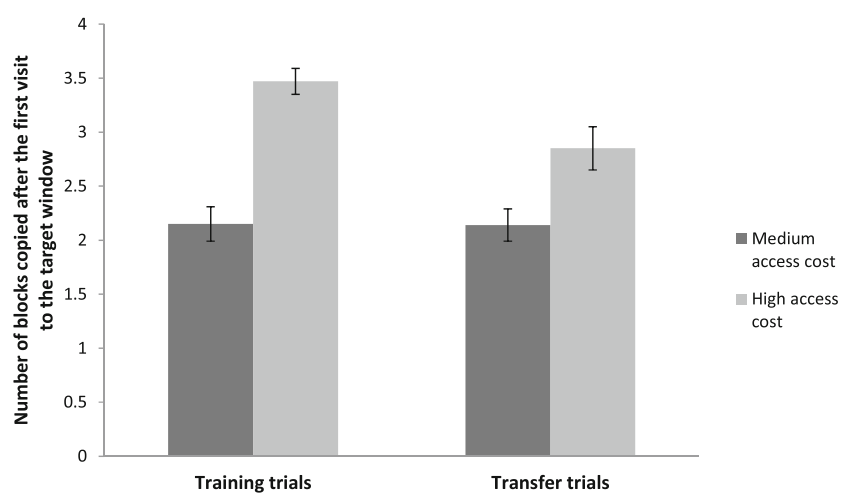

Fig. 2 The number of blocks copied correctly after the first visit to the target window across training and transfer trials (Experiment 1). Note. Error bars represent \pm 1 standard error significant reduction in the degree of memory-based strategy between the training and transfer environments $(p<.001)$, although these participants still maintained a higher memory-based strategy than those in the Medium access-cost condition in both training $(p<.001)$ and transfer environments $(p<.01)$. These results indicate that both previous training and the transfer environment contribute to the degree of memory-based strategy adopted in the transfer environment by participants who experienced High access-cost training. From the mean values we calculate that the High access-cost condition maintained $55 \%$ of their higher memory-based strategy from training when confronted with the transfer environment. Conversely, $45 \%$ of their high memorybased strategy induced during training was lost in the change between these two environments. Therefore both training and transfer environments contributed substantially to the degree of memory-based strategy deployed.

\section{Experiment 2}

The carryover effect between the training and transfer environments found in Experiment 1 may not occur when the costbenefit tradeoff of changing the degree of memory-based strategy is less attractive. In Experiment 2 the training and transfer conditions of Experiment 1 were reversed in order to investigate the influence of training in a medium access-cost environment on subsequent performance in a high access-cost transfer environment, which would normally be congruent with a higher memory-based strategy. We predicted that there will be little or no carry-over effect from the medium accesscost training for the following reasons. Experience in the training environment with no access time delay induces a lower memory-based strategy involving more visits to view and encode parts of the target pattern. However, if this strategy level is maintained during the high access-cost transfer environment, where the extra time delay is imposed, then experience of lockout time delays will increase considerably. In turn this 
will make the cost-benefit tradeoff of maintaining this lower degree of memory-based strategy unattractive. As a consequence, extrapolating from the soft constraints theory (Gray et al., 2006), we predict that the lower memory-based strategy adopted during training will be switched to a higher memorybased strategy in the transfer trials, because participants wish to avoid paying the cost of increased lockout time. Consequently we predict there will be no difference between the High and Medium access-cost training conditions in the transfer environment.

\section{Methods}

Participants

Twenty-six Cardiff University students participated and were randomly and equally assigned to one of two conditions. There were 20 women and six men with an age range of 18 20 years $(M=18.73, S D=.60)$.

\section{Materials}

The same BWT was used as in Experiment 1.

\section{Design}

A similar training and transfer design was used as in Experiment 1. Participants in the Medium access-cost training condition experienced 30 medium access-cost trials and those in the High access-cost training experienced the same number of high access-cost trials. Subsequently participants in both conditions experienced ten transfer trials in a high access-cost environment.

Procedure

The same procedure was used as in Experiment 1.

\section{Results and discussion}

During the High access-cost transfer phase there were no differences between the High and Medium access-cost conditions in the number of blocks moved correctly after the first target window visit, $t(24)=.03, p=.98, d=.01(M=3.56, S D$ $=.88$ and $M=3.55, S D=.53$ respectively), average time spent during the first visit to the target window, $t(24)=.70, p=.49, d$ $=.29(M=5.60, S D=2.09$ and $M=5.09, S D=1.59$ respectively), and average number of visits made to the target window, $t(24)=.77, p=.45, d=.31(M=3.51, S D=1.05$ and $M=$ $3.81, S D=.92$ respectively). Consequently, there is no evidence of any transfer effect from the Medium access-cost training when High access-cost transfer trials were encountered. Instead participants in the Medium access-cost training switched to a higher memory-based strategy on encountering the High access transfer trials and were indistinguishable in their performance from participants who received High access-cost training. Therefore the results from Experiments 1 and 2 suggest that the extent of any carry-over effects from previous training will be determined by the specific relationship between the training and transfer environments and the associated cost-benefit trade-off.

\section{Experiment 3}

The positive transfer effect of the high access-cost training environment to a medium access-cost environment, demonstrated in Experiment 1, is likely to depend upon the amount of training that is given because this variable strongly determines both the degree of learning and transfer in a variety of tasks (e.g., Crossman, 1959; Newell \& Rosenbloom, 1981; Provost, Johnson, Karayanidis, Brown, \& Heathcote, 2013). In Experiment 1, 30 training trials produced a substantial transfer effect, although Luchins (1942) and Lovett and Anderson (1996) only used five and eight trials, respectively, and still found strong effects of training. From the perspective of the theory of soft constraints (Gray et al., 2006), it is important to determine how much prior training in a high access-cost environment is necessary to influence transfer behavior in a medium access-cost transfer environment. Therefore, in Experiment 3, the number of high accesscost training trials was reduced in comparison to that provided in Experiment 1.

\section{Methods \\ Participants}

One hundred and four Cardiff University students participated in the study and were randomly assigned to one of four conditions. Given a reduction in training is likely to reduce the large effect sizes found in Experiment $1(f=.47-.79)$, the sample size for Experiment 3 was calculated to detect a medium to large effect size $(f=.25-.40)$ with power of .8 .

\section{Materials}

The materials were the same as in Experiment 1. 


\section{Design}

In the training phase, the number of high access-cost training trials, across four conditions, was $0,1,10$, and 20 (labeled 0High condition, i.e., a no-training control condition, 1High with one High access training trial, etc.). The transfer phase again consisted of ten medium access-cost trials, as in Experiment 1.

Procedure

The procedure was the same as that in Experiment 1, except that the experiment took 30-60 minutes to complete.

\section{Results and discussion}

Data from one participant in the $10 \mathrm{High}$ condition was not recorded due to equipment failure and two statistical outliers were replaced with the grand mean. During the transfer phase, participants in the 1,10 , and 20 High conditions copied the most blocks, spent more time in the target window, and made fewer visits to view the target pattern than those in the control condition (Table 2). One-way ANOVAs confirmed significant main effects of training condition on: the number of blocks copied correctly after the first visit to the target window, $F(3$, $99)=6.39, M S E=1.05, p<.001, f=.44$; average time spent in the target window during the first visit, $F(3,99)=6.23, M S E$ $=4.84, p=.001, f=.44$; and number of visits to the target window, $F(3,99)=12.96, M S E=3.72, p<.001, f=.63$. Bonferroni post-hoc comparisons revealed that participants in the 20High and 10High conditions copied more blocks than those in the 0High control condition $(p s<.01$ and $=.001$ respectively), although performance on this measure in the 1High condition was not significantly different to that of the control condition $(p=.22)$. However, participants in the 1,10 , and 20 High conditions spent more time in the target window during the first visit during transfer than the 0High control condition ( $p s<.05,<.001$, and $=.05$ respectively), and also made fewer visits to the target window during transfer trials than the 0High control condition $(p s<.05,<.001$, and $<.001$ respectively). Additionally, there were no significant differences among the 20High, 10High, and 1High training conditions on all three measures $(p s>.05)$. As in Experiment 1, there were strong positive relationships between the time spent encoding and planning in the target window during the first visit and the subsequent number of blocks copied correctly for the control $(r(26)=.70, p<.001)$, 1High $(r(26)=.78, p$ $<.001) 10 \mathrm{High}(r(25)=.64, p<.001)$, and 20High conditions $(r(26)=.61, p<.001)$.

Given the reductions in the amount of training given in comparison with Experiment 1, it was of interest whether any training conditions would be associated with some dissipation of the carry-over effects across the ten transfer trials. One-way ANOVAs on the number of blocks copied correctly after the first visit to the target window found no significant differences across transfer trials for the 1 High $F(6.79,169.85)$ $=2.04, M S E=3.29, p=.06$ (a Huynh-Feldt correction was applied due to violation of sphericity), 10High, $F(7.81$, $187.39)=.93, M S E=2.89, p=.49$ (again with a HuynhFeldt correction applied), and the 20High conditions, $F(9$, $225)=1.05, M S E=2.24, p=.40$. Surprisingly, and consistent with the results of Experiment 1, there is no evidence of a reduction of the effect of High access-cost training in the degree of memory-based strategy adopted by any condition across the transfer trials.

Overall these analyses have established a strong transfer effect from the two training conditions that involved 10 and 20 high access-cost training trials. These amounts of training mitigated to some extent the effect of the medium access-cost transfer environment. Surprisingly, the 1High training condition also had an effect on two of the three transfer strategy measures, indicating that even one training trial can have a carry-over effect and increase the degree of memory-based strategy adopted in the transfer environment. Participants in this condition spent more time encoding and planning in the target window during transfer trials in comparison with those in the control condition. However, given the effect of one training trial was not fully consistent, the aim of Experiment 4 was to adopt a more fine-grained approach and investigate

Table 2 The effect of training on transfer performance (Experiment 3)

\begin{tabular}{|c|c|c|c|c|c|}
\hline \multirow{2}{*}{$\begin{array}{l}\text { Measures of degree of memory-based strategy } \\
\text { (across ten transfer trials) }\end{array}$} & & \multicolumn{4}{|c|}{ Training condition (number of trials) } \\
\hline & & $\begin{array}{l}\text { 0High access-cost } \\
(\mathrm{n}=26)\end{array}$ & $\begin{array}{l}\text { 1High access-cost } \\
(n=26)\end{array}$ & $\begin{array}{l}\text { 10High access-cost } \\
(\mathrm{n}=25)\end{array}$ & $\begin{array}{l}\text { 20High access-cost } \\
(n=26)\end{array}$ \\
\hline $\begin{array}{l}\text { Number of blocks copied correctly after the first } \\
\text { visit to the target window }\end{array}$ & $\begin{array}{l}M \\
S D\end{array}$ & $\begin{array}{l}1.82 \\
0.73\end{array}$ & $\begin{array}{l}2.42 \\
1.39\end{array}$ & $\begin{array}{l}2.99 \\
0.96\end{array}$ & $\begin{array}{l}2.77 \\
0.90\end{array}$ \\
\hline $\begin{array}{l}\text { Time spent during the first visit to the target } \\
\text { window (s) }\end{array}$ & $\begin{array}{l}M \\
S D\end{array}$ & $\begin{array}{l}1.87 \\
0.86\end{array}$ & $\begin{array}{l}3.81 \\
3.06\end{array}$ & $\begin{array}{l}4.41 \\
2.68\end{array}$ & $\begin{array}{l}3.50 \\
1.49\end{array}$ \\
\hline Number of visits to the target window per trial & $\begin{array}{l}M \\
S D\end{array}$ & $\begin{array}{l}7.73 \\
2.54\end{array}$ & $\begin{array}{l}6.16 \\
2.29\end{array}$ & $\begin{array}{l}4.77 \\
1.12\end{array}$ & $\begin{array}{l}4.94 \\
1.35\end{array}$ \\
\hline
\end{tabular}


whether transfer effects could be detected after each occasion that an access delay was required to view the target pattern.

\section{Experiment 4}

Given some evidence in Experiment 3 that even one complete high access training trial was sufficiently potent to produce a positive transfer effect, Experiment 4 adopted a more microscopic intra-trial design rather than the inter-trial training and transfer designs of Experiments 1-3. The aim was to identify whether experiencing a high access-cost on one, two, or three visits to view the target pattern within one trial was sufficient to increase the degree of memory-based strategy subsequently used within that same trial. Experiment 4 therefore involved only one trial and was a particularly stringent test of intra-trial transfer because initial performance in a novel task is typically variable as participants "warm-up" and learn what is required of them (e.g., Hamilton \& Mola, 1953; Irion, 1948).

\section{Method}

\section{Participants}

One hundred and fifty-seven Cardiff University students participated and were randomly assigned to one of four conditions. There were 141 women and 16 men with an age range of $18-26$ years $(M=19.25, S D=1.29)$. Given that Experiment 4 focused on the fine-grained effects of up to three high access-cost visits, a small to medium effect size was predicted and sample size was calculated to detect this with power of .8 .

\section{Materials}

The same BWT was used as previously with the modification that thirteen different colored blocks were used in the $4 \times 4$ target grid in order to avoid any possible ceiling effect.

\section{Design}

Four between-participant conditions differed in terms of whether participants encountered a high $(\mathrm{H})$ or medium $(\mathrm{M})$ access cost on the first, second, and third visit to the target window. These conditions are labeled HHH, HHM, HMM, and MMM respectively. Thus the HHH condition experienced high access cost on each of the first three visits to the target window whereas HMM experienced this only on the first visit and medium access cost on the subsequent two visits. Two measures were used: the number of blocks correctly copied following each of the first three visits to the target window and the time spent viewing the target pattern during each of these visits.
Procedure

Participants received two practice trials with no access cost to familiarize them with the nature of the task and its interface requirements. Participants were given no practice with the medium or high access-cost conditions, which could have influenced the degree of memory-based strategy adopted in the subsequent trial.

\section{Results and discussion}

Data concerned one trial only and participants' performance during and after the first three visits to view the target pattern. The assumption of sphericity was violated with respect to time spent viewing the goal state, so a Huynh-Feldt correction was applied. In addition, seven outliers were present within the dataset and were replaced with the grand mean. Descriptive data are summarized in Table 3 and Fig. 3. It appears that experiencing one high access-cost visit has no effect, whereas experiencing two or three such visits increases the number of blocks copied correctly and therefore indicates a higher degree of memory-based strategy.

Analyses of the data involved two 4 (condition: HHH, HHM, HMM, MMM) $\times 3$ (first, second, third viewing of goal state) mixed ANOVAs. We were interested in possible differences among conditions at each target visit that would be reflected in interaction effects between conditions and target visits. There was a significant interaction for the number of blocks copied correctly between condition and target visit $F(6,306)=2.49$, $M S E=1.08, p<.025, f=.22$ (Fig. 3). There was also a significant interaction between condition and target visit on time spent viewing the target window $F(5.92,300.00)=5.81, M S E=2.40$, $p<.001, f=.34$. The simple effects within each of these interactions were analyzed and, for clarity of presentation, these are described below with respect to each target visit.

First target viewing If paying one high access cost of an extra 2.5-s lockout delay is sufficient to induce an increase in memory-based strategy, then participants in the $\mathrm{HHH}$, HHM, HMM conditions should deploy a higher memorybased strategy during and after the first target window visit than those in the MMM condition (Table 3). Bonferroni comparisons revealed that there were no differences among the four conditions in the number of blocks moved correctly after the first target visit ( $p s>.05)$ and the amount of time spent there ( $p s>.05)$, except for participants in the HMM condition who spent more time than those in the MMM condition, although this was only just significant $(p=.05)$. Consequently in five of six relevant comparisons among conditions, there is evidence that experiencing the access-cost delay on one 
Table 3 The effect of each access time delay on number of blocks copied correctly and time in the target window (Experiment 4)

\begin{tabular}{|c|c|c|c|c|c|c|}
\hline \multirow[t]{2}{*}{ Target visit } & \multirow[t]{2}{*}{ Measures of degree of memory-based strategy } & \multicolumn{5}{|c|}{$\begin{array}{l}\text { Sequence of high and medium access } \\
\text { viewings of target pattern }\end{array}$} \\
\hline & & & HHH (n=39) & HHM (n=39) & HMM $(n=39)$ & $\operatorname{MMM}(\mathrm{n}=40)$ \\
\hline \multirow[t]{4}{*}{ First } & Number of blocks copied correctly after first visit & $M$ & 1.21 & 1.05 & 1.15 & 0.98 \\
\hline & & $S D$ & 1.42 & 1.32 & 1.20 & 1.21 \\
\hline & Time spent in target window during first visit (s) & $M$ & 2.37 & 2.53 & 2.82 & 1.85 \\
\hline & & $S D$ & 1.69 & 1.79 & 1.71 & 1.79 \\
\hline \multirow[t]{4}{*}{ Second } & Number of blocks copied after second visit & $M$ & 2.17 & 1.67 & 1.26 & 1.20 \\
\hline & & $S D$ & 1.25 & 1.18 & 1.25 & 1.07 \\
\hline & Time spent in target window during second visit (s) & $M$ & 2.88 & 2.81 & 1.22 & 1.21 \\
\hline & & $S D$ & 1.35 & 1.25 & 1.33 & 1.23 \\
\hline \multirow[t]{4}{*}{ Third } & Number of blocks copied after third visit & $M$ & 2.46 & 1.69 & 1.49 & 1.15 \\
\hline & & $S D$ & 1.33 & 1.40 & 1.07 & 0.95 \\
\hline & Time spent in target window during third visit (s) & $M$ & 2.66 & 1.88 & 1.37 & 1.25 \\
\hline & & $S D$ & 1.28 & 1.70 & 1.23 & 1.34 \\
\hline
\end{tabular}

Note. $\mathrm{H}$ refers to high access cost of an extra $2.5 \mathrm{~s}$ in order to view the target pattern and $\mathrm{M}$ refers to medium access cost with no extra time delay

occasion only has no effect on the degree of memory-based strategy deployed.

Second target viewing If the payment of two high access costs are effective in inducing a more intensive memory-based strategy, then participants in the $\mathrm{HHH}$ and HHM conditions will exhibit a higher memory-based strategy than those in the HMM and MMM conditions with respect to performance during and after the second visit to view the goal state (Table 3). Bonferroni comparisons for the number of blocks copied correctly after the second viewing of the goal state revealed participants in the HHH condition copied more blocks than those in the HMM and MMM conditions ( $p s<.01$ ). However there were no differences in blocks copied correctly between participants in the HHM and both the HMM and MMM conditions ( $p s=.67$ and .42 respectively). Bonferroni comparisons of the time spent in the target window found that participants in both the HHH and HHM conditions spent more time viewing the target pattern than those in the HMM and MMM conditions $(p s<.001)$.

Consequently there is evidence in six of the eight relevant comparisons that experiencing two high access-cost lockouts resulted in an increase in the degree of memory-based strategy.

Third target viewing The final issue concerned condition $\mathrm{HHH}$ and whether paying a further high access cost when viewing the target pattern on the third occasion resulted in a higher memory-based strategy in comparison to the HHM condition that did not experience this extra lockout time. Results confirmed this as participants in the HHH condition copied more blocks correctly $(p<.05)$ and spent more time viewing the goal state $(p<.05)$ than those in the HHM condition. In addition, participants in the HHH condition would be expected to deploy a more memory-based strategy after the third target viewing than those in both the HMM and MMM conditions who only experienced one or no lockout time(s) respectively. This was confirmed with participants in the $\mathrm{HHH}$ condition copying significantly more blocks correctly after the third visit to the target window than those in HMM and MMM conditions ( $p s<.01$ and .001 respectively) and spending more time viewing the target pattern than those in the HMM and MMM conditions $(p s<.05)$.

In summary, there is strong evidence that experiencing a time delay of $2.5 \mathrm{~s}$ on one occasion only is insufficient to affect the degree of memory-based strategy, although paying this cost on two or three successive occasions results in an increase in the degree of memory-based strategy.

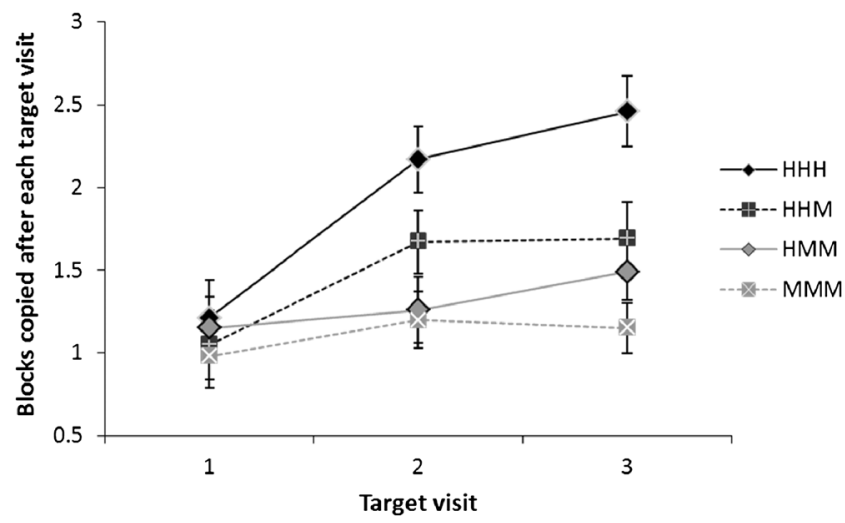

Fig. 3 The number of blocks copied correctly after each of the first three visits to view the target pattern (Experiment 4). Note. Error bars represent \pm 1 standard error 


\section{General discussion}

The aim of this study was to determine if previous training is an important factor to be considered by the theory of soft constraints (Gray et al., 2006). More specifically we examined whether and, if so, to what extent training with one access cost affected the degree of memory-based strategy adopted subsequently when performing the task with a different constraint. The results of Experiments 1 and 3 provide evidence that executing between one and 30 high access-cost training trials has a carry-over effect on subsequent performance of a set of medium access-cost transfer trials, which would normally be congruent with a lower degree of memory-based strategy. In Experiment 1, involving 30 training trials, the relative influence of the previous training to that of the transfer environment on the degree of memory-based strategy adopted during transfer performance was 55:45\% respectively. A reduction in the number of training trials to 20, ten, or one in Experiment 3 still produced a significant increase in the degree of memorybased strategy used in the transfer environment, indicating that there was no threshold number of training trials below which training did not influence performance. This was found (on two out of three measures) even when only one training trial was experienced before engaging in the transfer trials.

This result prompted a more fine-grained intra-trial examination of the effect of paying the access cost of an extra 2.5-s delay on the degree of memory-based strategy adopted within the same trial. Paying the time cost on one occasion only did not have a discernible effect on level of strategy adopted although paying this cost on two or three consecutive viewings of the target pattern did have an effect. Despite a couple of exceptions, the overwhelming evidence across two measures supports this conclusion. The results from Experiments 3 and 4 are somewhat surprising in that experiencing either one high access-cost training trial or high access cost on only two successive occasions within the same trial is sufficient to increase the subsequent degree of memory-based strategy. These findings add further corroboratory evidence in support of the theory of soft constraints. They demonstrate the potency of manipulating the access-cost constraint and the sensitivity of cognition in adapting to the current environment such that even fleeting experiences can leave their mark on subsequent strategy deployment. This finding is consistent with Logan's (1988) instance theory of memory, in which every performance of the same task is stored and increases the probability of these instances being recalled and influencing current performance, particularly when the task is novel and there is no existing algorithm to control behavior.

The results from Experiment 2 remind us that carry-over effects from training will not always occur and will depend upon the specific nature of the constraints experienced during training and transfer. The theory of soft constraints predicts that the cost-benefit tradeoff will become unattractive if a person persists with a lower memory-based strategy, developed in training, when confronted with a transfer environment with access time delays. Consequently, as our results demonstrate, a switch is made to deploy a higher memory-based strategy when access cost increases.

The soft constraints account of the importance of the constraints of the environment (Gray et al., 2006) together with accumulated evidence supporting this account (e.g., Fu \& Gray, 2000; Gray \& Fu, 2004; Morgan et al., 2009; Morgan \& Patrick, 2013; Morgan et al., 2013) contradict any simple minimal memory account of strategy deployment. The minimal memory account posits that people tend whenever possible to preserve cognitive resources in favor of less resource costly perceptual-motor based strategies (e.g., Ballard et al., 1995; Cary \& Carlson, 2001; Wilson, 2002). However, there is substantial evidence that when the cost (particularly time) of executing part of the task is increased, then participants will attempt to avoid or minimize that cost by extra cognitive processing. This is not only the case when information or goalstate access cost is increased (e.g., Fu \& Gray, 2000; Gray \& Fu, 2004; Gray et al., 2006; Waldron, Patrick, \& Duggan, 2011), as in the present study, but also when the implementation cost of executing a move is increased, which also results in increased planning (O'Hara \& Payne, 1998, 1999). In all of these studies participants have no alternative to experiencing the cost of a time delay when either the goal state is examined or the move is executed, and cognitive processing is adapted to minimize this cost (e.g., Anderson, 1990). However, a difference with the present study is that participants voluntarily continued to some extent with a higher memory-based strategy on the transfer trials than was necessary or congruent with the lower access cost in the transfer environment. Consequently, participants did not fully switch to minimize their internal processing as would be expected if the primary goal was to reduce the use of memory resources.

Some further issues and limitations of the present study need acknowledgment. First, we labeled some trials as "training" although no criterion of performance had to be achieved and there was no requirement for any particular strategy to be developed. Second, there are many factors associated with our research paradigm that are likely to have shaped our results, including the access-cost value, the seamless transition from training to transfer trials, and the number of transfer trials.

Our results have both theoretical and practical ramifications. They indicate that it is important that previous training be taken into account by the theory of soft constraints (Gray et al., 2006) because training can influence the degree of memory-based strategy adopted in a subsequent transfer environment in which the nature of the hard constraints differ. Further evidence in support of the theory of soft constraints is that the effect of only one high access-cost trial can be detected across at least ten subsequent trials in which there is no longer a high access-cost constraint. Also, performance can 
be influenced by as little as two occasions on which an access cost of 2.5-s is experienced.

Caution is needed in comparing the present study with general models of strategy selection, because both the nature of strategy and context differ so much from those of the present study. Lovett and Schunn's (1999) RCCL and Rieskamp and Otto's (2006) SSL models both emphasize the importance of reinforcement and previous success determining strategy selection. The present study is more in the tradition of the Adaptive Decision Maker (Payne, Bettman, \& Johnson, 1993), which adopts an adaptive cost-benefit approach. However, the model emphasizes that one key factor is the desire to conserve cognitive effort, although this is not borne out by the present findings, where participants deployed a more memory-based strategy in order to avoid a small time delay and continued to do so even when this access cost was no longer present in the task environment. Access cost is not mentioned by any of the general models of strategy selection and the fact that the introduction of even a fleeting access cost can affect the degree of memory-based strategy is a challenging finding for such models.

From a practical perspective we have two potential means of inducing a more memory-based strategy, when situations may benefit from such an approach, through either imposing an access cost during performance or developing some form of prior training regime. For example, a more memory-based problem solving strategy can be advantageous in exploratory learning (Sweller, 1988; Sweller and Levine, 1982) and in developing transfer between different variations of the same task (Vollmeyer, Burns, \& Holyoak, 1996). In addition, a more memory-based strategy can mitigate the effect of interruption during copying (Morgan et al., 2009) and problem solving (Morgan \& Patrick, 2013), improve the use of an interruption lag (Morgan et al., 2013), and improve the efficiency of planning in problem solving (Morgan et al., 2013). Also, in practical supervisory environments, operators are bombarded with an ever increasing array of information (Riley, Endsley, Bolstad, \& Cuevas, 2006) and they therefore may benefit from the development of a more memory-based processing strategy targeted at important or critical information.

Acknowledgments We are grateful to Anna Bowyer who collected some initial data for Experiment 3.

\section{References}

Allport, A., Styles, E. A., \& Hsieh, S. (1994). Shifting intentional set: Exploring the dynamic control of tasks. In C. Umilta \& M. Moscovitch (Eds.), Attention and performance XV (pp. 421-452). Cambridge: MIT Press.
Altmann, E. M., \& Gray, W. D. (2008). An integrated model of cognitive control in task switching. Psychological Review, 115, 602-639.

Anderson, J. R. (1987). Skill acquisition: Compilation of weak-method problem solutions. Psychological Review, 94, 192-210.

Anderson, J. R. (1990). The adaptive character of thought. Hillsdale: Erlbaum.

Anderson, J. R., Bothell, D., Byrne, M. D., Douglass, S., Lebiere, C., \& Qin, Y. (2004). An integrated theory of the mind. Psychological Review, 111(4), 1036-1060.

Anderson, J. R., \& Lebiere, C. (1998). The atomic components of thought. Mahwah: Erlbaum.

Ballard, D. H., Hayhoe, M. M., \& Pelz, J. B. (1995). Memory representations in natural tasks. Journal of Cognitive Neuroscience, 7(1), 66 80.

Campbell, J. I. D., \& Xue, Q. (2001). Cognitive arithmetic across cultures. Journal of Experimental Psychology: General, 130(2), 299 315.

Carroll, J. M., \& Rosson, M. B. (1987). Paradox of the active user. In J. M. Carroll (Ed.), Interfacing thought:Cognitive aspects of humancomputer interaction (pp. 80-111). Cambridge: MIT Press.

Cary, M., \& Carlson, R. A. (2001). Distributing working memory resources during problem solving. Journal of Experimental Psychology: Learning, Memory, and Cognition, 27, 836-848.

Chater, N., \& Oaksford, M. (1999). Ten years of the rational analysis of cognition. Trends in Cognitive Sciences, 3(2), 57-65.

Clawson, D. M., Healy, A. F., Ericsson, K. A., \& Bourne, L. E., Jr. (2001). Retention and transfer of Morse code reception skill by novices: Part-whole training. Journal of Experimental Psychology: Applied, 7, 129-142.

Crossman, E. R. F. W. (1959). A theory of the acquisition of speed-skill. Ergonomics, 2, 153-166.

De Corte, E. (2003). Transfer as the productive use of acquired knowledge, skills, and motivations. Current Directions in Psychological Science, 12, 142-146.

Faul, F., Erdfelder, E., Lang, A.-G., \& Buchner, A. (2007). G*Power 3: A flexible statistical power analysis program for the social, behavioral, and biomedical sciences. Behavior Research Methods, 39, 175-191.

Faul, F., Erdfelder, E., Buchner, A., \& Lang, A.-G. (2009). Statistical power analyses using $\mathrm{G}^{*}$ Power 3.1 : Tests for correlation and regression analyses. Behavior Research Methods, 41, 1149-1160.

Field, A. P. (2013). Discovering statistics using IBM statistics: And sex and drugs and rock ' $n$ ' roll (4th ed.). London: Sage.

Franks, J. J., Bilbrey, C. W., Lien, K. G., \& McNamara, T. P. (2000). Transfer-appropriate processing (TAP) and repetition priming. Memory \& Cognition, 28, 1140-1151.

Fu, W.-T., \& Gray, W. D. (2000). Memory versus perceptual-motor tradeoffs in a blocks world task. Proceedings of the 22nd annual conference of the Cognitive Science Society (pp. 154-159). Hillsdale, NJ: Erlbaum.

Fu, W.-T., \& Gray, W. D. (2004). Resolving the paradox of the active user: Stable suboptimal performance in interactive tasks. Cognitive Science, 28, 901-935.

Gick, M. L., \& Holyoak, K. J. (1987). The cognitive basis of knowledge transfer. In S. M. Cornier \& J. D. Hagman (Eds.), Transfer of learning: Contemporary research and applications (pp. 9-47). San Diego: Academic Press.

Gray, W. D., \& Boehm-Davis, D. A. (2000). Milliseconds matter: An introduction to microstrategies and their use in describing and predicting interactive behavior. Journal of Experimental Psychology: Applied, 6, 322-335.

Gray, W. D., \& Fu, W.-T. (2004). Soft constraints in interactive behavior: The case of ignoring perfect knowledge in-the-world for imperfect knowledge in-the-head. Cognitive Science, 28, 359-383.

Gray, W. D., Simms, C. R., Fu, W.-T., \& Schoelles, M. J. (2006). The soft constraints hypothesis. A rational analysis approach to resource 
allocation for interactive behavior. Psychological Review, 113, 461482.

Hamilton, C. E., \& Mola, W. R. (1953). Warm-up effect in human maze learning. Journal of Experimental Psychology, 45, 437-441.

Healy, A. F., Wohldmann, E. L., Sutton, E. M., \& Bourne, L. E., Jr. (2006). Specificity effects in training and transfer of speeded responses. Journal of Experimental Psychology: Learning, Memory, and Cognition, 32, 534-546.

Herrnstein, R. J., \& Vaughn, W. (1980). Melioration and behavioral allocation. In J. E. R. Staddon (Ed.), Limits to action: The allocation of behavior (pp. 143-176). New York: Academic Press.

Irion, A. L. (1948). The relation of "set" to retention. Psychological Review, 55, 336-341.

LeFevre, J., Bisanz, J., Daley, K. E., Buffone, L., Greenham, S. L., \& Sadesky, G. S. (1996). Multiple routes to solution of single-digit multiplication problems. Journal of Experimental Psychology: General, 125(3), 23.

Lemaire, P., \& Lecacheur, M. (2010). Strategy switch costs in arithmetic problem solving. Memory \& Cognition, 38(3), 322-332.

Loewenstein, G. F., Read, D., \& Baumeister, R. (2003). Time and decision: Economic and psychological perspectives on intertemporal choice. New York: Russell Sage Foundation.

Logan, G. D. (1988). Toward an instance theory of automatization. Psychological Review, 95, 492-527.

Lovett, M. C., \& Anderson, J. R. (1996). History of success and current context in problem solving: Combined influences on operator selection. Cognitive Psychology, 31, 168-217.

Lovett, M. C., \& Schunn, C. D. (1999). Task representations, strategy variability and base-rate neglect. Journal of Experimental Psychology: General, 128(2), 107-130.

Luchins, A. S. (1942). Mechanization in problem solving. Psychological Monographs, 54, 248.

Luchins, A. S., \& Luchins, E. H. (1959). Rigidity of behavior: A variational approach to the effect of Einstellung. Eugene: University of Oregon Books.

Luwel, K., Lemaire, P., \& Verschaffel, L. (2005). Children's strategies in numerosity judgment. Cognitive Development, 20 , 448-471.

Luwel, K., Schillemans, V., Onghena, P., \& Verschaffel, L. (2009). Does switching between strategies within the same task involve a cost? British Journal of Psychology, 100, 753-771.

Luwel, K., Verschaffel, L., Onghena, P., \& De Corte, E. (2003). Analyzing the adaptiveness of strategy choices using the choice/ no-choice method: The case of numerosity judgement. European Journal of Cognitive Psychology, 15, 511-537.

Monsell, S. (2003). Task switching. Trends in Cognitive Sciences, 7, 134 140

Morgan, P. L., \& Patrick, J. (2013). Paying the price works: Increasing goal access cost improves problem solving and mitigates the effect of interruption. Quarterly Journal of Experimental Psychology, 66(1), 160-178.

Morgan, P. L., Patrick, J., \& Tiley, L. (2013). Improving the effectiveness of an interruption lag by inducing a memory-based strategy. Acta Psychologica, 142(1), 87-95.

Morgan, P. L., Patrick, J., Waldron, S. M., King, S. L., \& Patrick, T. (2009). Improving memory after interruption: Exploiting soft constraints and manipulating information access cost. Journal of Experimental Psychology: Applied, 15(4), 291-306.
Morris, C. D., Bransford, J. D., \& Franks, J. J. (1977). Levels of processing versus transfer-appropriate processing. Journal of Verbal Learning and Verbal Behavior, 16, 519-533.

Newell, A., \& Rosenbloom, P. S. (1981). Mechanisms of skill acquisition and the law of practice. In J. R. Anderson (Ed.), Cognitive skills and their acquisition (pp. 1-55). Hillsdale: Erlbaum.

O'Hara, K. P., \& Payne, S. J. (1998). The effects of operator implementation cost on planfulness of problem solving and learning. Cognitive Psychology, 35, 34-70.

O'Hara, K. P., \& Payne, S. J. (1999). Planning and the user interface: The effects of lockout time and error recovery cost. International Journal of Human-Computer Studies, 50, 41-59.

Osgood, C. E. (1949). The similarity paradox in human learning: A resolution. Psychological Review, 56, 132-143.

Payne, J. W., Bettman, J. R., \& Johnson, E. J. (1993). The adaptive decision maker. New York: Cambridge University Press.

Provost, A., Johnson, B., Karayanidis, F., Brown, S. D. \& Heathcote, A. (2013). Two routes to expertise in mental rotation. Cognitive Science, 37,(7), 1321-1342

Reder, L. M. (1987). Strategy selection in question answering. Cognitive Psychology, 19, 90-138.

Reder, L. M., \& Klatzky, R. (1994). Transfer: Training for performance. In D. Druckman \& R. A. Bjork (Eds.), Learning, remembering, believing: Enhancing team and individual performance (pp. 25 56). Washington: National Academy Press.

Rieskamp, J., \& Otto, P. E. (2006). SSL: A theory of how people learn to select strategies. Journal of Experimental Psychology: General, $135,207-236$.

Riley, J. M., Endsley, M. R., Bolstad, C. A., \& Cuevas, H. M. (2006). Collaborative planning and situation awareness in army command and control. Ergonomics, 49, 1139-1153.

Roediger, H. L., III, Gallo, D. A., \& Geraci, L. (2002). Processing approaches to cognition: The impetus from the Levels-of-Processing framework. Memory, 10(5/6), 319-332.

Rogers, R. D., \& Monsell, S. (1995). Cost of a predictable switch between simple cognitive tasks. Journal of Experimental Psychology: General, 124, 207-231.

Siegler, R. S., \& Shipley, C. (1995). Variation, selection, and cognitive change. In T. Simon \& G. Halford (Eds.), Developing cognitive competence: New approaches to process modeling (pp. 31-76). Hillsdale: Erlbaum.

Sweller, J. (1988). Cognitive load during problem solving: Effects on learning. Cognitive Science, 12, 257-285.

Sweller, J., \& Levine, M. (1982). Effects of goal specificity on means ends analysis and learning. Journal of Experimental Psychology: Learning, Memory, and Cognition, 8, 463-474.

Thorndike, E. L., \& Woodworth, R. S. (1901). The influence of improvement in one mental function upon the efficiency of other functions. Psychological Review, 8, 247-261. 384-395, 553-564.

Vollmeyer, R., Burns, B. D., \& Holyoak, K. J. (1996). The impact of goal specificity on strategy use and the acquisition of problem structure. Cognitive Science, 20, 75-100.

Waldron, S. M., Patrick, J., \& Duggan, G. B. (2011). The influence of goal-state access cost on planning during problem solving. Quarterly Journal of Experimental Psychology, 64(3), 485-503.

Wilson, M. (2002). Six views of embodied cognition. Psychonomic Bulletin \& Review, 9, 625-636. 